\title{
Uso didáctico de las Tecnologías de Aprendizaje y Conocimiento (TAC), por parte de los docentes en educación básica secundaria y media
}

\author{
Didactic use of learning and knowledge technologies (TAC), by teachers in \\ secondary and secondary basic education
}

\author{
Diego Hernández \\ diegohv0003@gmail.com
}

Universidad Metropolitana de Educación, Ciencia y Tecnología, Colombia

\section{RESUMEN}

Esta investigación tuvo como propósito describir el uso didáctico de las Tac por parte de los docentes. Se hizo una investigación descriptiva, con diseño de campo transeccional contemporáneo univariable, y el abordaje fue cosmológico, exógeno y étic. La población estuvo conformada por 203 docentes y la muestra por 135 docentes de educación básica secundaria y media de las instituciones educativas públicas de Chiquinquirá, Boyacá, Colombia. Para la recolección de datos se diseñó una escala de uso didáctico de las Tac. El instrumento fue sometido a validez por expertos y validez estructural con índices entre 0,70 y 0,94 . La confiabilidad se calculó con la fórmula Alfa de Cronbach, y se obtuvieron índices entre 0,85 y 0,95 . Para el análisis de resultados se utilizaron porcentajes, mínimo, máximo y mediana. Se encontró que los docentes de básica secundaria y media de las instituciones educativas públicas del municipio de Chiquinquirá, tienen un bajo nivel de uso del tac en la planeación de los contenidos de las clases, igualmente sucede con la facilitación en el proceso de enseñanza aprendizaje. Se pudo establecer que los docentes con excelente uso didáctico del tac en el proceso de enseñanza aprendizaje son los docentes de tecnología e informática y de idioma extranjero inglés.

Palabras clave: Educación; interactividad; Tac; uso didáctico de las tac

\section{ABSTRACT}

The purpose of this research was to describe the didactic use of TAC by teachers. A descriptive investigation was made, with a univariable contemporary transectional field design, and the approach was cosmological, exogenous and ethical. The population was conformed by 203 teachers and the sample by 135 teachers of secondary and average basic education of the public educational institutions of Chiquinquirá, Boyacá, Colombia. For the data collection a scale of didactic use of the Tac was designed. The instrument was subjected to validity by experts and structural validity with indexes between 0.70 and 0.94 . Reliability was calculated with the Cronbach's Alpha formula, and indices between 0.85 and 0.95 were obtained. For the analysis of results, percentages, minimum, maximum and median were used. It was found that the secondary and middle school teachers of the public educational institutions of the municipality of Chiquinquirá, have a low level of use of the TAC in the planning of the contents of the classes, as well as the facilitation in the teaching-learning process. . It was established that the teachers with excellent didactic use of the TAC in the teaching-learning process were the teachers of technology and computer science and English foreign language.

Key words: Education; TAC; didactic use of TAC; interactivity 


\section{INTRODUCCIÓN}

Las Tac en el campo educativo han traído una serie de cambios, que obligan a que las partes implicadas en el proceso de enseñanza aprendizaje se adapten día a día a las nuevas exigencias de una sociedad globalizada. Además, la información y la comunicación fluyen a grandes velocidades, lo que conlleva a que las instituciones educativas, estudiantes, familias, secretarías de educación y, principalmente, los docentes deban involucrar las tecnologías educativas a la praxis pedagógica.

El docente, como uno de los actores fundamentales del proceso educativo al ser facilitador, guía y transmisor de conocimiento en el proceso de enseñanza, se convierte en uno de los pilares esenciales a partir de la necesidad de actualizarse frente al ritmo acelerado que imponen las tecnologías en los diferentes campos del conocimiento. Es por ello, que para mejorar la calidad educativa, primero se debe describir el estado actual de los docentes frente a cuatro eventos esenciales: su actitud frente a las Tac, el uso didáctico que hacen de las mismas, el nivel de conocimiento de las tecnologías educativas y el grado de interactividad en el aula.

Por tanto, este trabajo busca dar aportes para mejorar la enseñanza a través de las Tac en la educación básica secundaria y media colombiana, mediante el uso didáctico de éstas, como estrategia para motivar a los docentes hacia mejores prácticas educativas, $\mathrm{y}$ aprovechar el impacto de las Tac en la era digital. Esta investigación abordará temas referentes a los nuevos escenarios educativos para la docencia, el impacto en el entorno del estudiante, la nueva teoría conectivista, los diferentes modelos didácticos que enmarcan hoy día la educación, la actitud docente frente a las tac, el uso didáctico de las tac, la interactividad y el conocimiento que tienen los docentes sobre las tac.
La importancia de la educación en el siglo XXI radica en la necesidad de garantizarle al ser humano un aprendizaje permanente, y de mejorar las competencias que necesitará a lo largo de la vida, para que se realice desde lo personal, participe de la sociedad y logre finalmente, tener éxito en el mundo laboral (MEN, 2009).

En este sentido, la educación básica primaria y secundaria en Colombia están llamadas a propiciar una formación general, mediante el acceso, de manera crítica y creativa, al conocimiento científico, tecnológico, artístico y humanístico y de sus relaciones con la vida social y con la naturaleza, de manera tal que prepare al educando para los niveles superiores del proceso educativo y para su vinculación con la sociedad y el trabajo.

Sin embargo, la educación en Colombia atraviesa por varias dificultades, entre ellas se resalta el bajo nivel de calidad, la inequidad, la repitencia académica y la deserción escolar. En cuanto a la calidad, Colombia avanza en materia de calidad educativa, lo cual se ha visto reflejado en las diferentes pruebas nacionales e internacionales. En las pruebas Saber $11^{\circ}$ evaluadas por el ICFES en los años 2017 y 2016 se consiguió mantener el mismo nivel de logro. Se aumentó en 7 puntos al pasar de 250 puntos el promedio del año 2015 a 257 puntos en una escala de 500, en el año 2016. No obstante, a pesar de que el puntaje aumentó, todavía sigue siendo bajo, porque sólo dominan un poco más del $50 \%$ de las competencias.

A pesar de la leve mejoría que se evidencia, existen aún causas que denotan un bajo nivel de calidad educativo: la desigualdad entre la educación pública y privada, donde los resultados en las pruebas nacionales organizadas por el ICFES, muestran mejor nivel de preparación en los estudiantes de los colegios privados, falta de mejor infraestructura tecnológica, mayor recursos de parte del es 
tado para material didáctico en las aulas de clase, mayor motivación a los docentes, ya que su labor no es reconocida ni premiada, y falta de capacitación a los docentes mediante el manejo y uso de las tecnologías para el aprendizaje y conocimiento (TAC).

Por otro lado, en Colombia aún se observa el predominio de una educación tradicional, en la que la memoria es el pilar de ésta, y desafortunadamente, aún se encuentran en las aulas docentes que enseñan únicamente con marcador y tablero. La educación tradicional presenta desventajas, como hacer énfasis en lo memorístico, la inactividad, la falta de interactividad, la no formación de competencias sociales, la falta motivación, la falta de realismo, el generar aburrimiento, entre otros, ya que está dirigida solo a la obtención de buenos resultados en calificaciones, más no a un aprendizaje significativo.

Es por ello que la tecnología se convierte en un factor esencial en la educación, que ha traído consigo cambios en los métodos de enseñanza e incluso en la formación y actualización docente. Su importancia en la educación radica en permitir la construcción didáctica y la consolidación de un aprendizaje significativo con la ayuda de las herramientas tecnológicas. De esa manera se pueden superar las limitantes de la enseñanza tradicional, permite un aprendizaje social y colaborativo, y permite generar una educación transformadora y adaptable a la sociedad (Díaz, 2013).

La presencia de la tecnología tiene implicaciones en todos los ámbitos de la vida, hoy día las noticias y la información circulan a gran velocidad, incluso los conocimientos se encuentran a tan solo un clic. La sociedad experimenta cambios en todas las esferas, desde la comunicación hasta en la manera que se interactúa con los demás, de hecho, a través de las redes sociales se rompen las barreras de la distancia.
Incluso la presencia de las tecnologías de apoyo profesional (Tap), han cambiado la forma de trabajar: los arquitectos han cambiado el lápiz y papel por programas como AutoCAD, los diseñadores ahora usan la tecnología del renderizado con software como $3 \mathrm{dMax}$ que les da la ventaja de poder corregir en tiempo real los diseños e imprimir en cualquier instante. La medicina también evoluciona gracias a la tecnología, actualmente se usa con mayor frecuencia las ecografías $3 \mathrm{~d}$ y $4 \mathrm{~d}$ para observar los fetos en movimiento y detectar posibles malformaciones, que para este caso sería más complejo con una simple radiografía o ecografía tradicional. Es por esto que la educación también debe evolucionar, tal cual como lo han hecho otras profesiones. Para el caso de la educación, existen múltiples tecnologías, pero las que más pueden contribuir en este contexto son las tecnologías de aprendizaje y conocimiento (Tac). Las Tac incorporan las tecnologías de información y comunicación (Tic) y todas las herramientas tecnológicas que se pueden integrar a la educación.

Ante esto la tecnología educativa, cuenta con recursos que ofrecen grandes ventajas, al utilizarlas eficazmente se logra estimular tanto al docente como al estudiante, para crear espacios interactivos donde se puede lograr de forma activa la participación, la acción, el realismo la inmersión y la multisensorialidad, Los estudiantes entran en contacto con sus profesores, sobre todo cuando se trabaja en proyectos, que son parte de una enseñanza situada en el contexto, y promueven el aprendizaje significativo (Ortega, 2014). Pero para conseguirlo es necesario cambiar el pensamiento que se tiene sobre las Tac, es decir, la actitud y la forma de enfrentar las tecnologías en educación. No puede haber cambio en la educación al integrar la tecnología si la metodología sigue siendo la misma. 
Es por las razones antes expuestas que los docentes deben incorporar las tecnologías a la didáctica, porque con las potencialidades de herramientas, se puede lograr un cambio significativo en la educación y un mejoramiento de la calidad de la misma. Sin embargo, los docentes muestran todavía resistencia hacia el uso didáctico de las tecnologías que podrían enriquecer y contribuir a mejorar el aprendizaje de los estudiantes (Cabero, 2007). Una de las problemáticas es la actitud docente frente a las tecnologías, diversas investigaciones señalan que la actitud de los docentes es negativa. Generalmente, los resultados obtenidos muestran que los docentes manifiestan temor hacia ellas, sienten dificultades en el uso dentro del aula y un aumento significativo de su trabajo (Valdés y otros, 2012). También la falta de conocimiento de parte de los docentes en la conceptualización, uso y manejo de las Tac, puede generar, en parte, la resistencia a usar las tecnologías en el campo educativo. Si un docente no sabe manejar y enfrentarse a las herramientas tecnológicas y a internet, seguirá realizando su praxis pedagógica de manera tradicional, y condenará a los estudiantes a aprendizajes memorísticos con falta de inmersión y realismo.

Es por ello que el MEN hace esfuerzos para mejorar el nivel de conocimiento de los docentes a través de capacitaciones, donde se ha trabajado en marcos que buscan mejorar de manera significativa la formación docente y apropiación educativa con las tecnologías, muestra de ello fue el plan decenal de educación 2006-2016 que trata de garantizar el acceso, uso y capacitación docente de las tecnologías en los ámbitos de enseñanzaaprendizaje, y se han esforzado por diseñar módulos y programas para la formación de docentes, dotar de infraestructura a las instituciones educativas, garantizar acceso de co- nectividad y construir currículos que integran las tecnologías en el ámbito educativo.

De las razones antes expuestas se deriva la importancia de generar acciones para superar esas dificultades, que contribuirán significativamente a mejorar la práctica docente, su actitud y conocimiento hacia las Tac, que finalmente se verán reflejadas en una mejor calidad educativa. Por tanto, esta investigación fue dirigida a describir el uso que hacen, los docentes del municipio de Chiquinquirá, Boyacá, Colombia, de las herramientas tecnológicas en la didáctica escolar.

\section{Bases teóricas}

Según UNESCO (2013), las escuelas se enfrentan a la necesidad de innovar en los métodos pedagógicos si desean convocar y ser inspiradoras para las nuevas generaciones de jóvenes. Ya que, a través de nuevos currículos, modelos educativos, didácticas educativas entre otras, también se puede mejorar la calidad educativa y motivar a los estudiantes a seguir sus estudios.

La enseñanza pasiva, se suma a los inconvenientes educativos, donde el docente es el protagonista, ya que este se encarga de desarrollar las clases a través de la comunicación verbal, y con herramientas como el libro, el tablero y el marcador, lo que convierte a los estudiantes en receptores, sentados en sus puestos, con una mínima participación que se limita a sólo tomar apuntes sobre lo que el docente explica, para luego memorizarlo (Fundación Comillas, 2018). Para Arranz (2017), algunas de las desventajas de esta educación memorística son: que genera un aprendizaje básico, mecánico, los contenidos se relacionan arbitrariamente, los datos se recuerdan, la mayoría de las veces, a corto plazo, la información se olvida fácilmente, no permite analizar la información, y no motiva al estudiante a seguir aprendiendo. 
Una tendencia importante en la educación del tercer milenio es la incorporación de la tecnología a los procesos de enseñanza y de aprendizaje (UNESCO, 2009). Una de las corrientes que más se ha centrado en el uso de la tecnología es el conectivismo. Para Siemens (2004), el conectivismo es la integración de principios explorados por las teorías de caos, redes, complejidad y auto-organización; el aprendizaje es un proceso que ocurre al interior de ambientes difusos de elementos centrales cambiantes que no están por completo bajo control del individuo, que puede residir fuera de nosotros (al interior de una organización o una base de datos), está enfocado en conectar conjuntos de información especializada, y las conexiones que nos permiten aprender más tienen mayor importancia que nuestro estado actual de conocimiento. El

Modelo didáctico-tecnológico se relaciona con el conectivismo, ya que este modelo se combina la preocupación de transmitir el conocimiento acumulado con el uso de metodologías activas. Existe preocupación por la teoría y la práctica, de manera conjunta (Fernández y Madrid, 2010).

La didáctica tecnológica que para McLoughlin y Lee (2008), es la pedagogía 2.0, está definida por:

- Contenido diverso: Micro unidades que ofrecen diversas perspectivas a los estudiantes y recursos generados por ellos mismos.

- Currículum dinámico: Programaciones no fijas sino dinámicas, abiertas a la negociación y la aportación del estudiante.

- Comunicación multifacética, entre pares, abierta, con múltiplos tipos de medios para conseguir claridad y relevancia.

- Proceso iterativo: Procesos reflexivos integrados y contextualizados que son dinámicos y basados en la investigación y la actuación.
- Recursos múltiples: fuentes, tanto formales como informales, que son de alcance global y ricas en diversidad de medios.

- Ayudas en red: Andamiajes y apoyo para que los estudiantes reciban ayuda de pares, profesores, expertos y comunidades.

- Tareas de aprendizaje personalizadas: Tareas auténticas, conducidas y diseñadas por los propios estudiantes, relacionados con su experiencia.

Existen diferentes tipos de tecnología, pero las vinculadas a la educación se pueden clasificar en tecnologías de información y comunicación (Tic), tecnologías de aprendizaje y conocimiento (Tac), y tecnologías de apoyo profesional (Tap).

Las Tecnologías de información y comunicación (Tic), son el grupo de tecnologías destinadas para que los usuarios puedan tener acceso, producción, tratamiento y comunicación de la información que es presentada a través de diferentes medios textuales, imágenes, sonidos entre otros. El uso de las Tic en la educación puede ampliar el acceso que tienen las personas a oportunidades de aprendizaje, mejorar la calidad de la educación, a través de métodos avanzados de enseñanza, así como impulsar la reforma de los sistemas educativos (UNESCO, 2009).

Si bien en un principio se utilizó la expresión Tic para referirse a todas tecnologías de información y comunicación, y también a las educativas, más recientemente se viene utilizando la expresión tac, para aludir a aquellas tecnologías que se ocupan del aprendizaje y la construcción de conocimiento. La idea central de las Tac, parte de que tanto el docente como estudiante puedan aprender de manera permanente fuera de las Instituciones de Educación (Lozano, 2011).

Otro tipo de tecnologías son las de apoyo profesional (Tap). Las tecnologías de apoyo profesional son aquellas que aportan herramientas de trabajo que específicas que cada 
profesional puede utilizar en su disciplina. Por ejemplo, los arquitectos utilizan AutoCAD para realizar planos, las ecografías han evolucionado a 3D y 4D, de igual forma los contadores públicos utilizan softwares contables. Las Tic, las Tac y las Tap, cada día ganan más presencia en el mundo, y particularmente en el contexto educativo, como medios para orientar la educación en todos los ciclos.

Lozano (2011) aclara que algunos autores han hecho una comparación entre Tac y Tic. Aquellos que prefieren utilizar la expresión Tac aseguran que el concepto de Tic es excesivamente informático, instrumentalista y poco motivador frente a los requerimientos que necesitan los profesores y estudiantes actuales, y que pueden aprender a utilizar. Las Tac son herramientas informáticas dirigidas especialmente a potencias el aprendizaje y la generación de conocimiento, y existe una gran variedad de ellas. Algunas son los entornos de aprendizaje, foros, blogs, Web 5.0, Wikis, redes sociales, simulaciones, video tutoriales y App educativas, entre otras

Bakia, Murphy, Anderson y Trinidad (2011), consideran que, mediante las clases en compañía del docente y uso apropiado de las tecnologías, se puede impactar la calidad de la educación y contribuir a reforzar el proceso de aprendizaje del estudiante para generar un mejor desempeño en sus competencias, especialmente cuando se interactúa con videos, sitios webs, y simulaciones, entre otros.

La forma cómo la didáctica se apoya en la tecnología puede denominarse uso didáctico de las tac. El uso didáctico de las Tac se define como la acción que realiza el docente en su contexto, en la cual se vale de los diferentes recursos tecnológicos disponibles para desarrollar proceso educativo y propiciar un mejor aprendizaje.

Hay que ser conscientes de los procesos y retos que supone el uso didáctico de las tecnologías, que involucran nuevos escenarios y ambientes educativos en el marco de la virtualidad y la velocidad de transmisión de la información y que dan lugar campos virtuales y escenarios 2.0, 3.0 y 5.0 donde se desarrollan ciertas acciones educativas (Cabero, 2007).

Para Santiago, Caballero, Gómez y Domínguez (2013), el uso de las tecnologías ofrece un mundo de alternativas didácticas con el fin de mejorar los procesos de enseñanza aprendizaje. Permite acercarse al estudiante en la comunicación y facilita la orientación de contenidos. Coll (2011 como se citó en Bautista, 2017), concreta cinco agrupaciones de los posibles usos de las Tac:

- Consultar, seleccionar, administrar la información, exponerla y fomentar el aprendizaje a través de recursos multimedia.

- Para la praxis docente, planificación, búsqueda de información, actualización de contenidos con objeto de enseñanza, registro de calificaciones, presentaciones con dibujos, esquemas, multimedia, textos, hipermedia.

- Para la interrelación, se efectúa de manera más dinámica la comunicación entre los actores educativos, donde expresan sus ideas, solicitan información, orientan contenidos y ejercicios didácticos e intercambian vivencias.

- Para fortalecer actividades didácticas: dar explicaciones, aclarar dudas, para corregir, realizar seguimiento a las actividades.

- Para configurar entornos de trabajo y aprendizaje: como videotutoriales, plataformas como Moodle, redes de intercambio de información entre pares y la puesta en marcha de actividades en línea.

En todo caso el uso de las tecnologías es una vía para que el docente cumpla de mejor manera las responsabilidades que se le atribuyen. Esta actividad de incorporar la tecnología a los procesos educativos se denomina 
uso didáctico de las Tac. El uso didáctico de las Tac, abarca las siguientes dimensiones: planificación, motivación, facilitación y evaluación.

La planificación de las Tac se refiere a las acciones que el docente lleva a cabo para organizar, buscar, estructurar y programar el uso de las Tac para la administración de los contenidos y actividades de la clase, de acuerdo a los objetivos de la asignatura. Según Ramos (2000 como fue citado por Rivero y Moscoso, 2013) la planificación en educación es la acción para resolver situaciones a través de estrategias previas con el fin de lograr objetivos, metas y finalidades, para el control y la toma de decisiones, en función del tiempo y los recursos disponibles.

La motivación hacia las Tac se refiere a las acciones que el docente lleva a cabo para generar interés y agrado hacia el uso de las Tac por parte de los estudiantes, durante el proceso de enseñanza.

Para Valenzuela (2015), la motivación tiene que ver con la activación de recursos cognitivos para aprender lo que el docente propone como aprendizaje, y no sólo con la intención del estudiante de querer realizar una tarea. En este sentido, la motivación tiene que ver, en términos generales, con el valor de la tarea y con el sentimiento de competencia frente a ella, pero también, con los motivos que dan sentido a la activación de dichos recursos cognitivos para el mejoramiento del aprendizaje.

La facilitación basada en las Tac se refiere a las actividades que puede generar el docente desde la práctica educativa hacia los estudiantes, en las cuales hace uso de las Tac para facilitar el proceso de aprendizaje en los estudiantes, a partir de la presentación de contenidos, la realización de ejercicios y el uso de diversas técnicas de enseñanza. Para Quintero (2008), la facilitación implica definir una secuencia de acciones centradas en el aprendiz, que hacen más efectivo el proceso de aprendizaje a través de la implementación de actividades y ejercicios en el aula. La facilitación es un apoyo provisto por el docente para hacer posible o más fácil determinadas acciones, para potenciar y acelerar los procesos que permiten los logros el aprendizaje.

La evaluación basada en las Tac se refiere a la valoración de los avances y los resultados del proceso de enseñanza, a partir de evidencias que garanticen una educación pertinente y significativa para el estudiante, y relevante para la sociedad, con apoyo de las diversas tecnologías de aprendizaje y conocimiento.

Para incorporar el uso de las Tac al aula es necesario que los jóvenes tengan conocimiento de las mismas y que estén dispuestos a utilizarlas. Sin embargo, para Francesc (2014), los jóvenes colombianos al terminar secundaria hacen un uso moderado de las tecnologías educativas, aun cuando en ocasiones el uso del computador lo destinan más hacia el ocio que a tareas educativas. De todas maneras, esto puede beneficiar otras competencias, como, por ejemplo, el aprendizaje autónomo y el uso de estrategias meta cognitivas, que a la postre se relacionan directamente con el logro escolar, aun cuando esto pudiera sonar paradójico.

Por otra parte, Santiago, Caballero, Gómez y Domínguez (2013), piensan que incorporar las Tac en el aula de clase implica un apoyo a los docentes, para que utilicen en su praxis pedagógica tecnologías como los recursos multimedia que facilitan el aprendizaje de nuevos conocimientos. En este sentido, Pinzón (2017) realizó una investigación en la Institución educativa rural El Cerro, del municipio de Chía, Cundinamarca, donde concluyó que la institución cuenta con recursos tecnológicos propicios para mejorar el proceso de enseñanza y de aprendizaje. Sin embargo, los recursos son poco utilizados por los docentes 
para apoyar el proceso de enseñanza. Además, los docentes manifiestan y reconocen la importancia del uso de las tecnologías como estrategia didáctica en el proceso de enseñanza, no obstante, reconocen que son poco usadas en su ámbito educativo, y desaprovechan el potencial de estas herramientas para motivarse y motivar a los estudiantes.

Esto lleva a pensar en que los docentes se enfrentan a la necesidad de obtener nuevas competencias, para contribuir a que los estudiantes fortalezcan y/o adquieran nuevas destrezas, habilidades y actitudes. Es fundamental entre tanto, que en su nuevo rol, el docente descubra sus motivaciones, inquietudes e intereses (Viñals y Cuenca, 2016).

La presencia de las Tac en las clases no asegura la adquisición de conocimientos, pero puede promover mecanismos de interactividad en el aula, que estimulen los procesos de enseñanza y aprendizaje.

\section{MÉTODO}

Se trabajó desde una visión holística de la ciencia dado que se partió de una noción integrativa del conocimiento. Se utilizó el mé- todo holopráxico que consiste en el paso organizado por cada uno de los estadios de la espiral holística, hasta llegar al estadio propio del objetivo general, que en este caso es el estadio descriptivo.

Esta investigación se llevó a cabo bajo la forma de una investigación descriptiva, mediante un diseño de campo. En cuanto a la temporalidad, el diseño fue transeccional contemporáneo. Dado el número de eventos, el diseño fue univariable. El abordaje fue cosmológico, exógeno y étic

El contexto fue Chiquinquirá, municipio colombiano, localizado en la provincia de occidente del departamento de Boyacá. La población de estudio estuvo conformada por todos los docentes de educación básica secundaria y media de las instituciones educativas públicas de Chiquinquirá (Boyacá). La población total fue de 203 docentes. Para la presente investigación se realizó un muestreo probabilístico estratificado, para garantizar que todas las instituciones tuviesen representación en la muestra. La muestra quedó conformada como se muestra en el cuadro 1.

Cuadro 1. Muestra estratificada por institución

\begin{tabular}{lccc}
\hline \multicolumn{1}{c}{ Institución Educativa } & $\begin{array}{c}\text { Total } \\
\text { Docentes }\end{array}$ & $\begin{array}{c}\text { \% Docentes } \\
\text { en la población }\end{array}$ & $\begin{array}{c}\text { Muestra } \\
\text { por estrato }\end{array}$ \\
\hline Los Comuneros & 9 & 4,43 & 6 \\
Liceo José Joaquín Casas & 40 & 19,70 & 27 \\
Técnica Industrial Julio Flórez & 44 & 21,67 & 29 \\
$\begin{array}{l}\text { Normal Superior Sor Josefa } \\
\text { del Castillo y Guevara }\end{array}$ & 45 & 22,17 & 30 \\
Técnica Pio Alberto Peña & 29 & 14,29 & 19 \\
Técnico Comercial Sagrado Corazón de Jesús & 36 & 17,73 & 24 \\
\hline \multicolumn{1}{c}{ Total } & $\mathbf{2 0 3}$ & $\mathbf{1 0 0}$ & $\mathbf{1 3 5}$ \\
\hline
\end{tabular}

Fuente: Elaboración propia a partir de datos obtenidos en las instituciones de la población.

Para la recolección de los datos acerca del uso didáctico de las Tac, se utilizó como técnica la encuesta, y como instrumento una escala Likert denominada Escala de uso didáctico de las Tac, que constó de 52 ítems con 3 alternativas de respuesta: nunca, a veces $y$ frecuentemente. Cada ítem puntuaba de 0 a 2 , y la escala dio un puntaje máximo de 104 puntos. Para su interpretación se utilizó una escala transformada de 0 a 50 puntos, los ítems se agruparon en 4 sinergias; planifica- 
ción con 19 ítems, motivación con 9 ítems, facilitación con 14 ítems y evaluación con 10.

En la validez por expertos de la Escala de uso didáctico de las Tac, los resultados arrojaron un índice de validez 0,94 . También se aplicó la técnica de validez estructural, que se basó en la correlación de las dimensiones del evento de estudio con la totalidad del evento, igualmente de cada ítem con cada dimensión y con el total. Los valores se ubicaron entre 0,86 y 0,91 . Para determinar la confiabilidad de la Escala de uso didáctico de las Tac, se utilizó el coeficiente alfa de Cronbach. Se tomó como muestra piloto 68 encuestas de los docentes estudiados, para calcular la confiabilidad tanto global, como por sinergias. La confiabilidad total de la prueba fue de 0,95 . La confiabilidad para las sinergias del evento uso de las Tac, fue de 0.85 para planificación, 0,86 para facilitación, 0,74 para motivación y 0,74 para evaluación.

\section{RESULTADOS}

Para el análisis del uso didáctico de las Tac que hacen los docentes en su labor educativa, se transformaron los puntajes brutos arrojados por la Escala de Uso didáctico de las Tac, y se obtuvo, para cada docente, un puntaje transformado en una escala de 0 a 50 puntos. Se calcularon la mediana, el mínimo, el máximo y los cuartiles, y se elaboró el gráfico de caja. Se utilizó la mediana porque el nivel de medición del evento fue ordinal. Para interpretar los puntajes obtenidos se utilizó el cuadro 2.

Cuadro 2. Intervalos y categorías del uso didáctico de las Tac

\begin{tabular}{cl}
\hline Intervalo & \multicolumn{1}{c}{ Categoría } \\
\hline $0-9,9$ & Muy insuficiente \\
$10-19,9$ & Deficiente \\
$20-29,9$ & Regular \\
$30-39,9$ & Suficiente \\
$40-50$ & Excelente \\
\hline
\end{tabular}

Fuente: Elaboración propia

Según el cuadro 3, el grupo obtuvo una mediana de 18,75 puntos sobre 50 puntos, que al ser comparada con el cuadro 2 , se ubicó en la categoría deficiente. Esto significó que, en términos generales, el uso didáctico que hacían de las Tac los docentes era mínimo dentro del proceso educativo. 
Cuadro 3. Mediana del grupo de docentes en uso didáctico de las Tac

\begin{tabular}{lrr}
\hline Mediana & & 18,750000 \\
Mínimo & & 4,3269 \\
Máximo & & 46,1538 \\
Percentiles & 25 & 13,461538 \\
& 50 & 18,750000 \\
& 75 & 26,442308 \\
\hline
\end{tabular}

Fuente: Elaboración propia a partir del instrumento aplicado a los docentes.

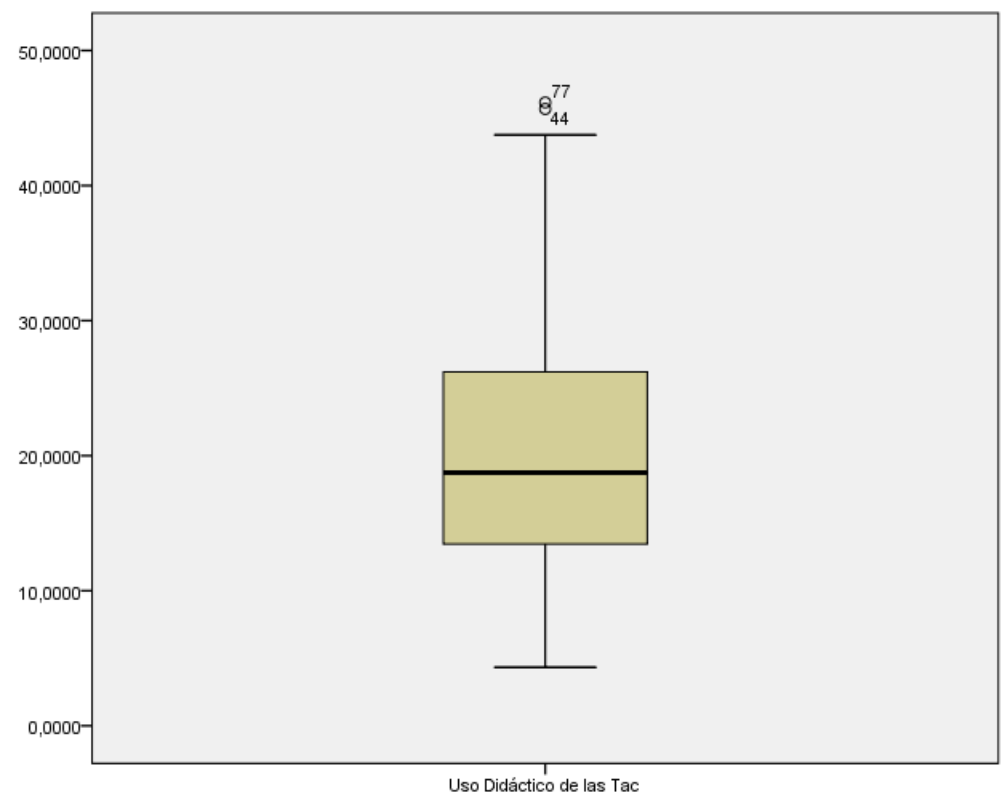

Gráfico 1. Mediana de los docentes en uso didáctico de las Tac.

Fuente: Elaboración propia a partir del instrumento aplicado a los docentes.

El grafico 1 mostró que el puntaje mínimo fue de 4,32 puntos, ubicado en la categoría muy deficiente, y el puntaje máximo fue de 46,15 puntos, ubicado en la categoría excelente. El grupo fue muy disperso, porque hay puntajes a lo largo de toda la escala de 0 a 50 . Los puntajes por encima de la mediana fueron más heterogéneos que los que se mostraron por debajo, lo que significó que en ese sector el grupo presentaba características más dispersas. Se encontraron casos en todas las categorías.

También se encontraron dos casos excepcionales con puntajes muy por encima de su grupo: el caso 77, docente de género femenino, del área de idioma extranjero inglés, de la institución educativa Técnico Industrial
Julio Flórez, cuya edad oscilaba entre los 35 y 40 años, y el caso 44, docente de género masculino, licenciado en educación industrial, a cargo del área de tecnología e informática en la institución educativa Técnico Comercial Sagrado Corazón de Jesús cuya edad oscilaba entre los 40 y 45 años. El caso 77 pertenecía a un docente del área de tecnología e informática, lo que resultó congruente ya que esta asignatura debía involucrar en mayor medida las tecnologías, y el caso 44 pertenecía a una docente de inglés: es claro que utilizaba con mayor frecuencia las tecnologías, ya que para facilitar el aprendizaje del inglés se trabajaba con diferentes medios multimedia.

En cuanto a los porcentajes de uso didáctico, se observó que entre las categorías defi- 
ciente y muy deficiente se ubicó un 55,55\% de los docentes, es decir, más de la mitad de la muestra total no incorporaban las tecnologías en la didáctica con sus estudiantes. Mientras que los docentes que tenían un buen uso didáctico de las tac, eran tan solo el 11,85\%, ubicados en las categorías suficiente, el $8,89 \%$, y en excelente, el 2,96. Lo que signifi- caba que muy pocos docentes incorporaban las diferentes tecnologías en la didáctica con sus estudiantes.

El uso didáctico de las Tac estaba conformado por cuatro sinergias: planificación, motivación, facilitación y evaluación. A continuación, se presenta el análisis de cada sinergia.

Cuadro 4. Medianas del grupo de docentes en las sinergias de uso didáctico de las Tac

\begin{tabular}{llrrrr}
\hline & & $\begin{array}{c}\text { Dimensión } \\
\text { Planificación }\end{array}$ & $\begin{array}{c}\text { Dimensión } \\
\text { Motivación }\end{array}$ & $\begin{array}{c}\text { Dimensión } \\
\text { Facilitación }\end{array}$ & $\begin{array}{c}\text { Dimensión } \\
\text { Evaluación }\end{array}$ \\
\hline $\mathrm{N}$ & Válidos & 135 & 135 & 135 & 135 \\
Mediana & & 23,68 & 19,44 & 14,28 & 15,00 \\
Mínimo & 6,57 &, 00 & 1,78 &, 00 \\
Máximo & 48,68 & 47,22 & 46,42 & 47,50 \\
Percentiles & 25 & 18,42 & 13,88 & 8,92 & 10,00 \\
& 50 & 23,68 & 19,44 & 14,28 & 15,00 \\
& 75 & 30,26 & 27,77 & 21,42 & 22,50 \\
\hline
\end{tabular}

Fuente: Elaboración propia a partir del instrumento aplicado a los docentes.

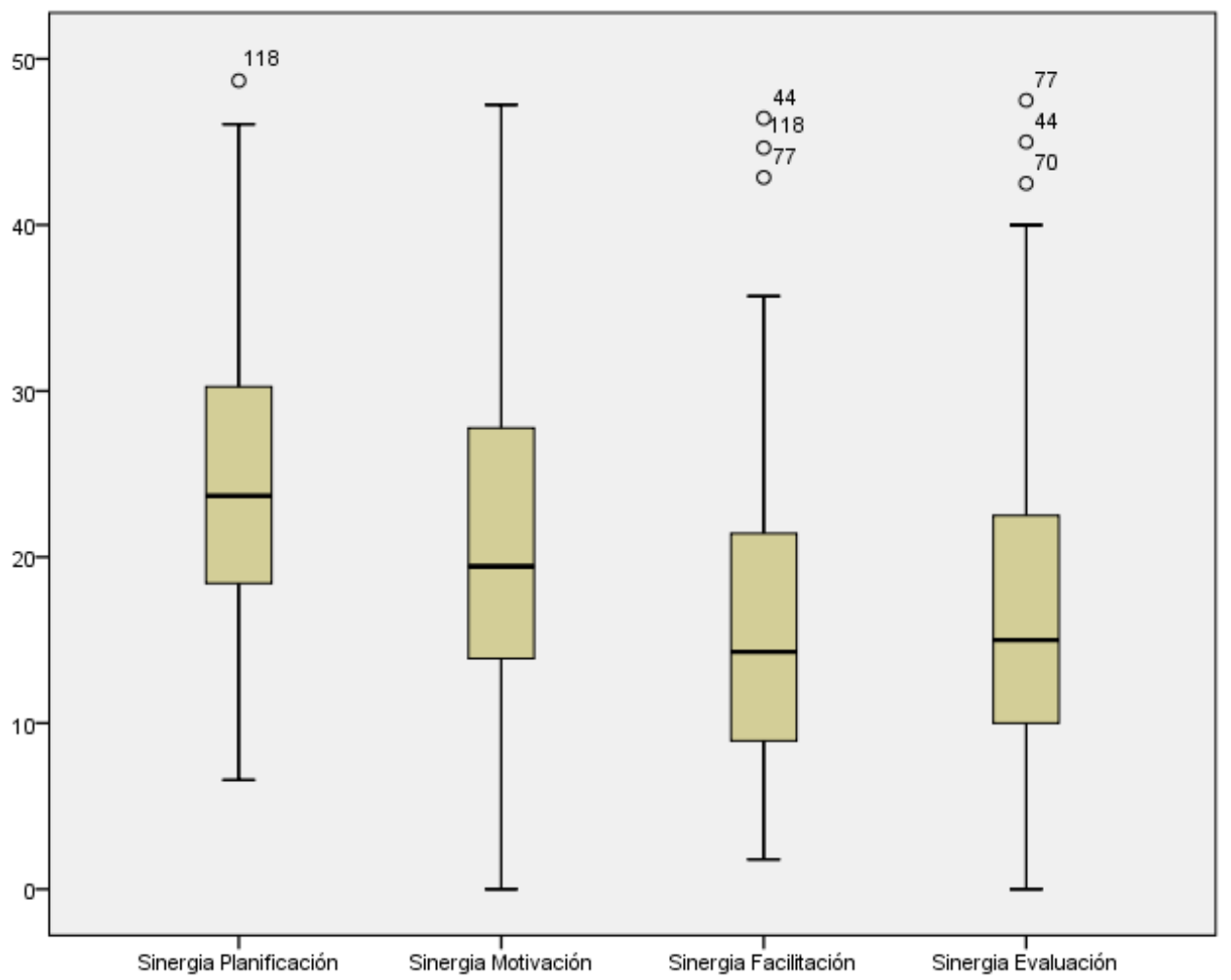

Gráfico 2. Mediana de los docentes en las dimensiones de uso didáctico las Tac Fuente: Elaboración propia a partir del instrumento aplicado a los docentes. 
En el cuadro 4 y gráfico 2 se aprecia que las distribuciones de todas las medianas fueron muy heterogéneas, y que los docentes obtuvieron puntajes desde muy bajos hasta muy altos. Sin embargo, el mejor puntaje fue el de planificación, que se ubicó en la categoría regular, y resulta interesante que la mediana de facilitación se ubicó en la categoría deficiente, por debajo de planificación, lo que significó que los docentes planificaban para incorporar la tecnología a las clases, pero lue- go, en el desarrollo de las clases esas tecnologías no se aplicaban.

Los resultados de la sinergia planificación se muestran en el cuadro 5 donde se observó una mediana de 23,68 sobre 50 puntos, que al ser comparada con el cuadro 2 de intervalos y categorías de interpretación se ubicó en la categoría regular. Esto indicaba, que el grupo de docentes planificaba sólo en ciertas ocasiones el uso didáctico de las Tac para trabajar los contenidos temáticos.

Cuadro 5. Mediana del grupo de docentes en la sinergia planificación

\begin{tabular}{lrr}
\hline Mediana & & 23,6842 \\
Mínimo & & 6,58 \\
Máximo & & 48,68 \\
Percentiles & 25 & 18,4211 \\
& 50 & 23,6842 \\
& 75 & 30,2632 \\
\hline
\end{tabular}

Fuente: Elaboración propia a partir del instrumento aplicado a los docentes.

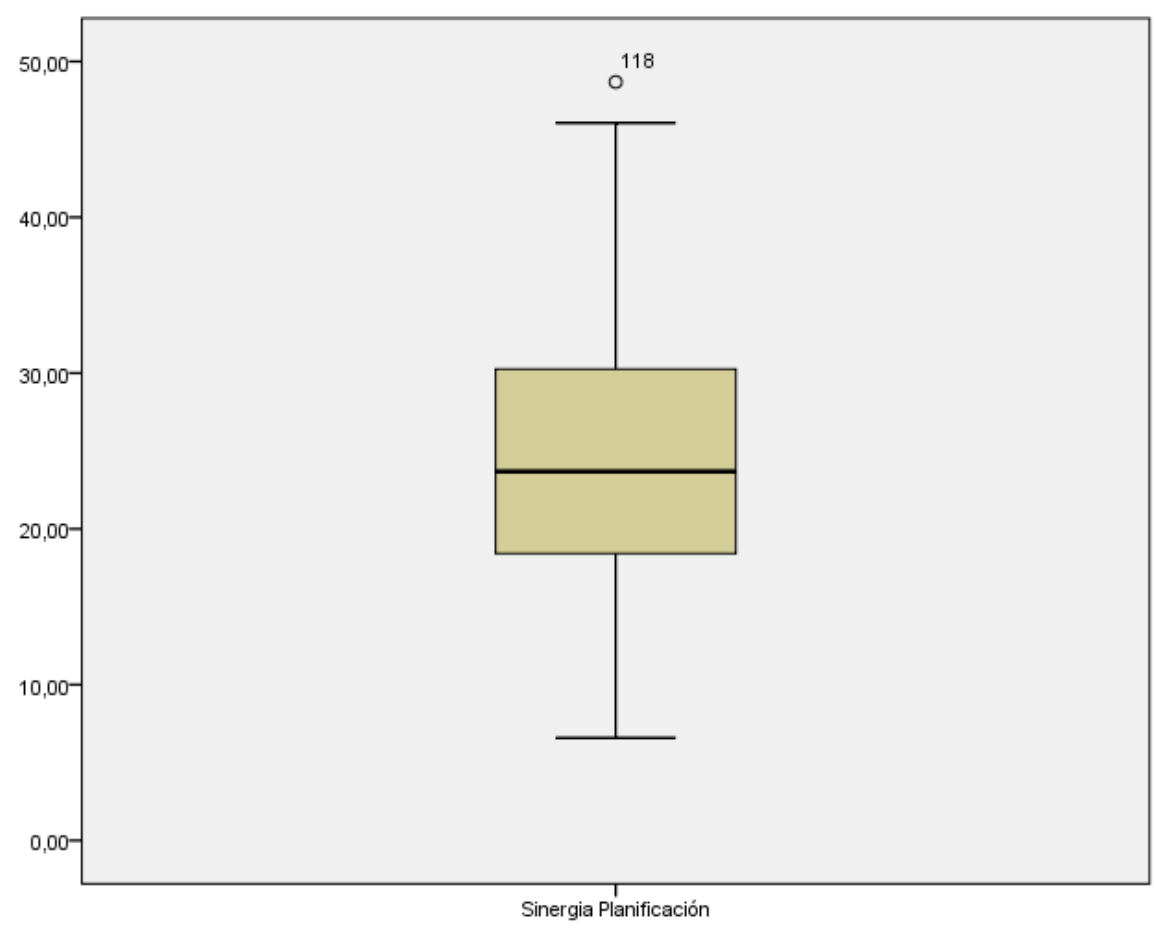

Gráfico 3. Mediana de los docentes en la sinergia planificación, de uso didáctico las Tac.

Fuente: Elaboración propia a partir del instrumento aplicado a los docentes. 
El gráfico 3, mostró que el puntaje mínimo fue de 6,58 puntos, y se ubicó en la categoría muy deficiente, y el puntaje máximo fue de 48,68 puntos sobre 50 de la escala transformada, ubicado en la categoría excelente. Los puntajes por encima de la mediana fueron un poco más heterogéneos que los que se encontraban por debajo, lo que significaba que en ese sector el grupo presentaba características más dispersas.

Se encontraron casos en todas las categorías, desde la categoría muy deficiente hasta la categoría excelente. Se presentó un solo caso excepcional, con un puntaje muy por encima de su grupo, el caso 118 docente de género masculino, del área de pedagogía de la institución educativa Normal superior, Magister en Educación, cuya edad oscilaba entre los 30 y 35 años. El caso 118 era un docente de pedagogía, que debía orientar a los futuros normalistas en la manera correcta de preparar los contenidos y actividades, lo que posibilitaba que trabajara de manera más continua con tecnologías.

En cuanto a los porcentajes de la sinergia planificación, se observó que entre las categorías deficiente y muy deficiente se encontraba un $37,03 \%$, de los docentes. Lo que significaba que más de un tercio del número de docentes de la muestra total tenían tendencia a no usar las Tac en la preparación de los contenidos. Por otra parte, un 36,30\% preparaba los contenidos incorporando las Tac en algunas ocasiones. Por último, entre las categorías suficiente y excelente, el $26,67 \%$ de los docentes incorporaban las herramientas tecnológicas para fortalecer los contenidos a desarrollar en el aula.

Dentro de las fortalezas en el proceso de planeación de clase, las que tenían un mayor impacto fueron:

- Buscaban en internet imágenes para fortalecer la temática a explicar (99\%).
- Utilizaban motores de búsqueda para conseguir información (97\%).

- Para preparar clases consultaban información en bases de datos, bibliotecas, índices a través de internet (98\%).

- Elaboraban talleres en Word (94\%).

En las fortalezas registradas existía una inclinación a buscar información por medio de internet para preparar las clases y usaban herramientas básicas como procesadores de textos. En cuanto a las debilidades en el momento de la planeación de clases con las Tac se encontró:

- No construían apps educativas para colocar contenidos dinámicos (72\%).

- Nunca diseñaban elementos con software asistido por computador (51\%).

- No diseñaban video tutoriales para fortalecer los temas explicados (59\%).

- Nunca programaban actividades en plataformas (62\%).

- No diseñaban elementos o materiales para alojarlos en la plataforma (53\%).

- Nunca elaboraban blogs para compartir explicaciones de los temas (56\%).

Los docentes en su gran mayoría no usaban las tecnologías emergentes en los últimos años en la preparación de los planeamientos de clase, ya que no utilizaban blogs, plataformas educativas, apps, video tutoriales ni software que podrían mejorar significativamente la preparación de contenidos y actividades del docente.

Los resultados de la sinergia motivación se muestran en el cuadro 6, donde se observó una mediana de 19,44 sobre 50 puntos, que al ser comparada con el cuadro 2 de interpretación se ubicó en la categoría deficiente. Esto indicaba que el grupo de docentes de la población presentaba claras falencias al querer persuadir y motivar a los estudiantes en la consecución de los logros mediante la incorporación de las Tac. 
Cuadro 6. Mediana del grupo de docentes en la sinergia motivación

\begin{tabular}{lrr}
\hline Mediana & & 19,4444 \\
Mínimo & &, 00 \\
Máximo & & 47,22 \\
Percentiles & 25 & 13,8889 \\
& 50 & 19,4444 \\
& 75 & 27,7778
\end{tabular}

Fuente: Elaboración propia a partir del instrumento aplicado a los docentes

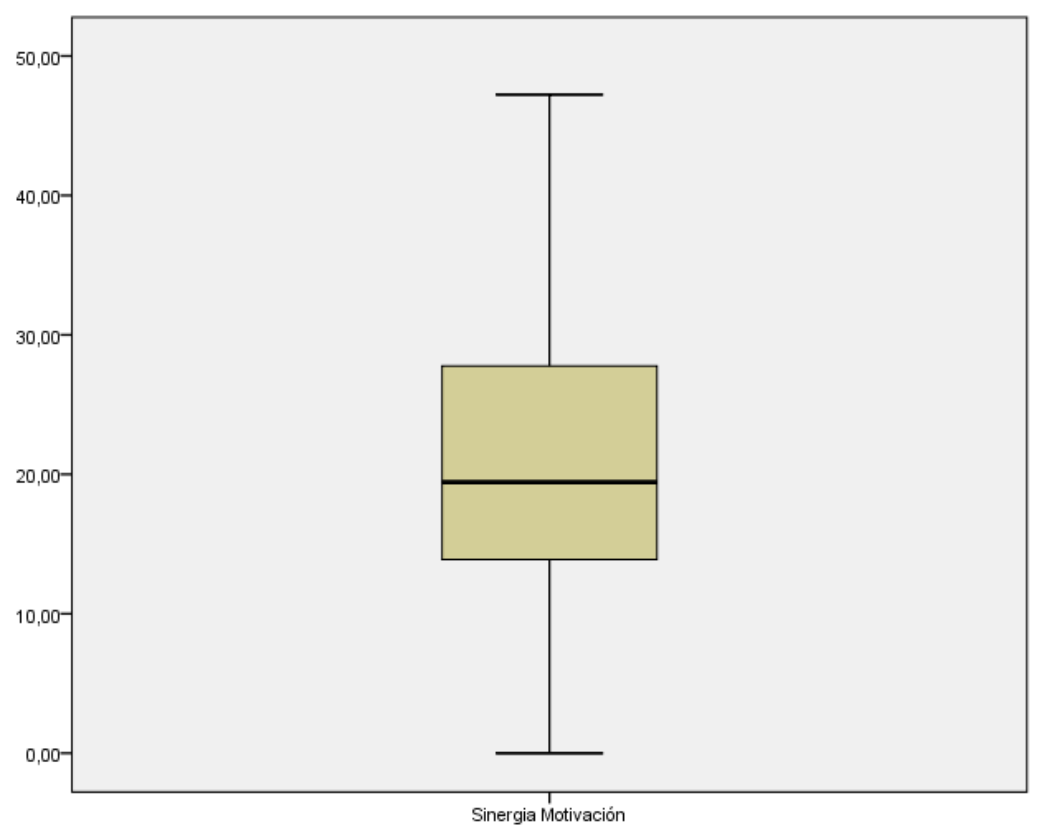

Gráfico 4. Mediana de los docentes en la sinergia motivación, de uso didáctico las Tac.

Fuente: Elaboración propia a partir del instrumento aplicado a los docentes

El grafico 4 mostró que, para la dimensión motivación, el puntaje mínimo fue de 0,00 puntos y el puntaje máximo fue de 47,22 puntos sobre 50 de la escala transformada. Los puntajes por encima de la mediana fueron un poco más heterogéneos que los que se mostraban por debajo, lo que significaba que en ese sector el grupo presentaba características más dispersas, aunque el grupo en general era bastante heterogéneo. Se encontraron casos en todas las categorías, lo que demostró que el grupo era muy heterogéneo. No se encontraron casos atípicos.

En cuanto a los porcentajes de la dimensión motivación, se observó que entre las ca- tegorías deficiente y muy deficiente se ubicó un $52,59 \%$ de los docentes. Lo que significaba que un poco más de la mitad de los docentes de la muestra total, les faltaba involucrar las Tac para motivar a los estudiantes a alcanzar los logros. Entre las categorías suficiente y excelente se obtuvo que el 18,51\% de los docentes utilizaban las Tac para guiar y motivar a los estudiantes a la consecución de los logros.

Dentro de las fortalezas, como es el proceso de guiar y motivar a los estudiantes a la adquisición de conocimientos por medio de las Tac, la sumatoria de porcentajes que tuvo 
un mayor impacto entre frecuentemente y a veces, fue:

- Implementaban internet en el desarrollo de la clase $(80 \%)$.

- Animaban a sus estudiantes para que usen el computador (96\%).

- Fomentaban la interacción a través del uso del computador (94\%).

Los docentes tenían tendencias a utilizar tecnologías estáticas, es decir, que tenían un nivel bajo de interactividad. Les faltaba incorporar tecnologías que permitieran clases más didácticas y dinámicas que motivaran a sus estudiantes a desarrollar y adquirir nuevas competencias en las áreas fundamentales del sistema escolar. En cuanto a las debilidades que se evidenciaron de parte de los docentes para motivar a sus estudiantes mediante el uso de las Tac se encontraron los siguientes aspectos:

- No usaban el correo electrónico para dar reconocimiento a los estudiantes (64\%).
- No usaban redes sociales para dar reconocimiento a estudiantes destacados (61\%).

- No estimulaban a sus estudiantes a trabajar con la plataforma institucional (55\%).

- No usaban las simulaciones, para hacer clases más atractivas (52\%).

Se pudo apreciar que los docentes presentaban dificultades para incorporar tecnologías que podían fortalecer la comunicación por medio de debates o foros a través de redes sociales o plataformas.

Los resultados de la sinergia facilitación se mostraron en el cuadro $\mathrm{N}^{\circ} 7$, donde se observó una mediana de 14,28 puntos sobre 50 puntos, que al ser comparada con el cuadro $\mathrm{N}^{\circ} 2$ de intervalos y categorías de interpretación se ubicó en la categoría deficiente. Lo cual indicó, que el grupo de docentes de la población analizada les faltaba implementar actividades donde se involucren las tecnologías en el aula de clase.

Cuadro 7. Mediana del grupo de docentes en la sinergia facilitación

\begin{tabular}{lrr}
\hline Mediana & & 14,2857 \\
Mínimo & & 1,79 \\
Máximo & & 46,43 \\
Percentiles & 25 & 8,9286 \\
& 50 & 14,2857 \\
& 75 & 21,4286 \\
\hline
\end{tabular}

Fuente: Elaboración propia a partir del instrumento aplicado a los docentes.

El grafico 5 mostró el puntaje mínimo de 1,79 puntos y el puntaje máximo fue de 46,43 puntos sobre 50 de la escala transformada. Se mostró que los puntajes por encima de la me- diana eran más heterogéneos que los que se mostraban por debajo, lo que significaba que en ese sector el grupo presentaba características más dispersas. 


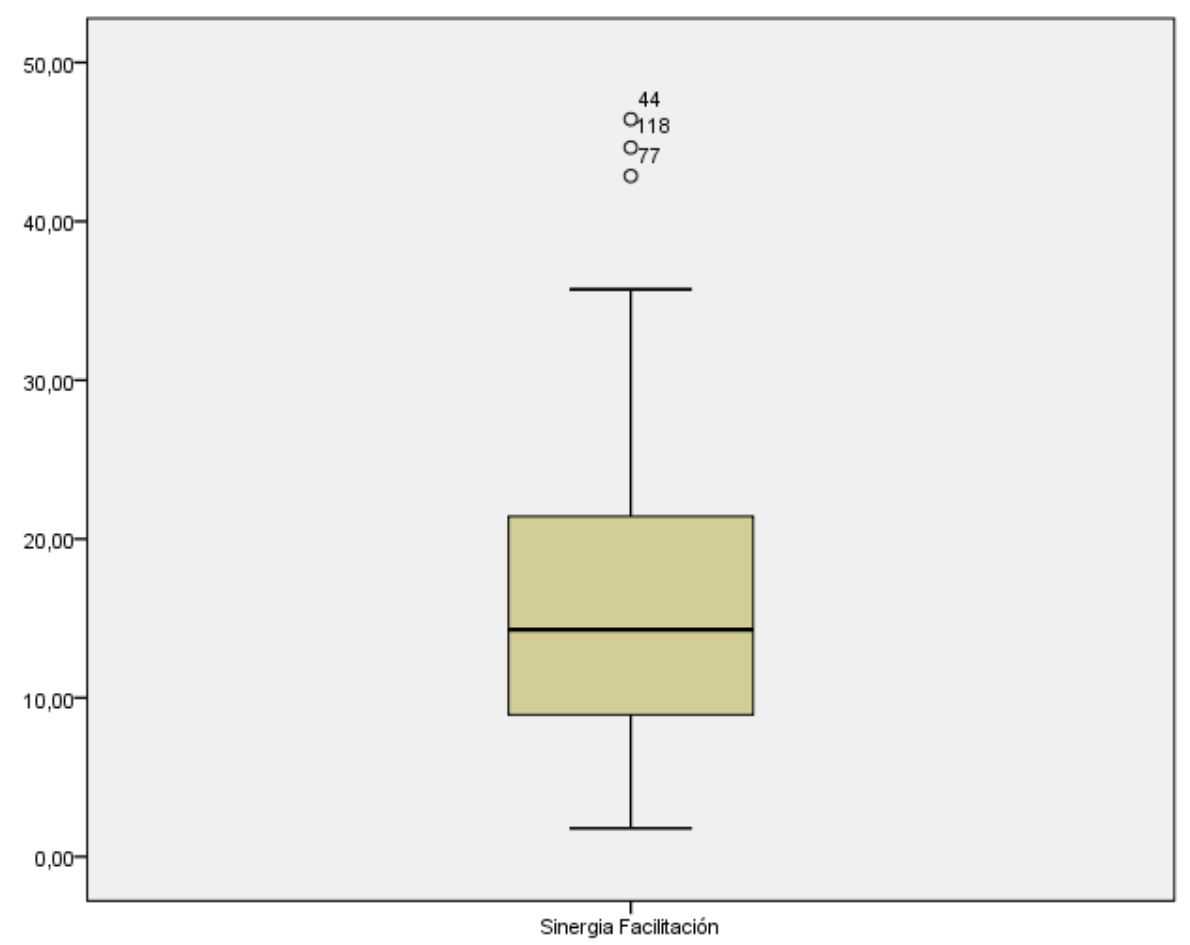

Gráfico 5. Mediana de los docentes en la dimensión facilitación, de uso didáctico las Tac Fuente: Elaboración propia a partir del instrumento aplicado a los docentes.

En cuanto a los porcentajes de la dimensión facilitación, se observó que un $71.11 \%$, de los docentes se ubicó entre las categorías deficiente y muy deficiente. Lo que significó que una gran parte del número de docentes de la muestra total les faltaba incorporar actividades que involucran las Tac en el proceso de enseñanza aprendizaje.

Dentro de las fortalezas en las actividades y apoyo a los contenidos explicados a partir de las Tac, las que tienen un mayor impacto en la sumatoria de porcentajes entre frecuentemente y a veces, son:

- Apoyan la explicación con actividades de consulta grupales en internet (84\%).

- Presentan y transmiten información por medio de Power Point (81\%).

- Desarrollan actividades desde el portal Colombia Aprende (70\%).

Como se pudo observar, los docentes que incorporaban dentro del desarrollo de sus contenidos y actividades a sus estudiantes, tenían un buen potencial en el uso de internet para actividades de consulta y apoyaban sus explicaciones por medio de tecnologías básicas como la presentación de diapositivas, pero les faltaba incorporar tecnologías más recientes dentro de los procesos educativos. En cuanto a las debilidades para proyectar actividades a partir de las Tac se encontró que:

- Poco retroalimentaban las temáticas desarrollando ejercicios desde un blog (62\%).

- Se les dificultaba realizar debates en wikis (76\%).

- No realizaban debates desde redes sociales (67\%).

- Con muy poca frecuencia realizaban presentaciones desde la plataforma para orientar las competencias a desarrollar (63\%).

- No asignaban tareas por medio del correo electrónico (53\%). 
- Poco utilizaban la realidad aumentada para potenciar habilidades cognitivas (58\%).

Se pudo apreciar que a los docentes se les dificultaba usar tecnologías en el desarrollo de actividades junto a sus estudiantes, para mejorar la comunicación e interactividad entre docente-estudiante por medio de wikis, blogs, redes sociales e incluso el correo electrónico para compartir información. Además, tecnologías actuales como realidad aumentada no eran incluidas dentro del proceso de enseñanza aprendizaje.

Los resultados de la sinergia evaluación se mostraron en el cuadro 8 donde se observó una mediana de 15,00 puntos sobre 50 , que al ser comparada con el cuadro 2 se ubicó en la categoría deficiente. Lo cual indicó, que el grupo de docentes no involucraban las tac en los procesos evaluativos a sus estudiantes.

Cuadro 8. Mediana del grupo de docentes en la sinergia evaluación

\begin{tabular}{lrr}
\hline Mediana & & 15,0000 \\
Mínimo & &, 00 \\
Máximo & & 47,50 \\
Percentiles & 25 & 10,0000 \\
& 50 & 15,0000 \\
& 75 & 22,5000 \\
\hline
\end{tabular}

Fuente: Elaboración propia a partir del instrumento aplicado a los docentes.

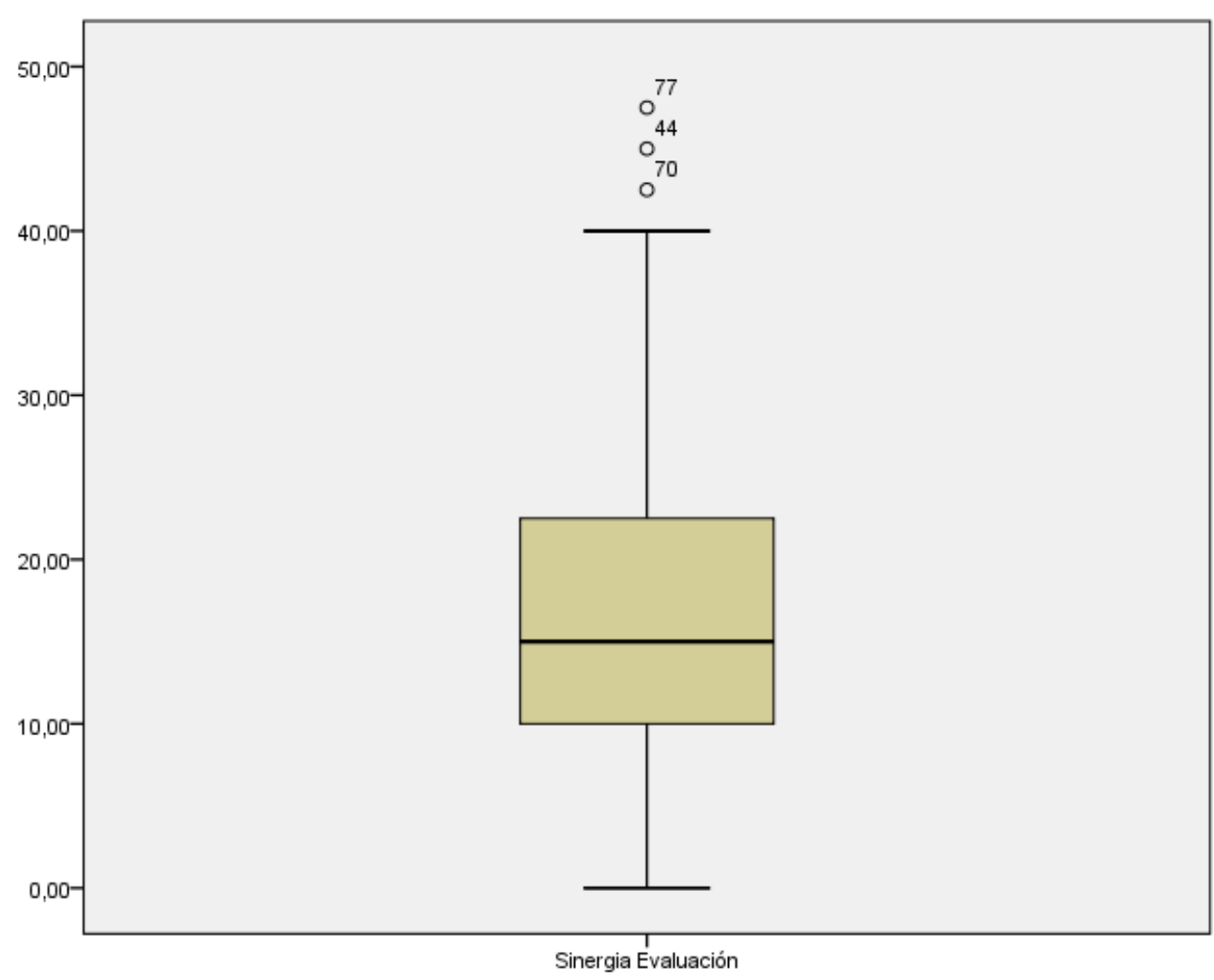

Gráfico 6. Mediana de los docentes en la dimensión evaluación, de uso didáctico las Tac Fuente: Elaboración propia a partir del instrumento aplicado a los docentes. 
El grafico 6, mostró el puntaje mínimo de 0,00 puntos, y el puntaje máximo de 47,50 puntos sobre 50 de la escala transformada. El grupo en general era bastante heterogéneo pues se encontraron casos en todas las categorías, es decir, desde la categoría muy deficiente hasta la categoría excelente. También se encontraron casos atípicos con puntajes muy por encima de su grupo: los casos $77 \mathrm{y}$ 44 eran los mismos con puntajes superiores en las dimensiones anteriores. Por otra parte, el caso 70 era un docente de género masculino, del área de tecnología e informática de la institución educativa Técnico Industrial Julio Flórez, cuya edad oscilaba entre los 35 y 40 años.

En cuanto a los porcentajes de la sinergia, se observó que entre las categorías deficiente y muy deficiente se ubicó un 60\%, de los docentes, lo que representó que a gran parte de ellos les faltaba incorporar las tecnologías en los procesos de evaluación de sus estudiantes. Dentro de las fortalezas de los docentes en la manera como evalúan a los estudiantes y registran las calificaciones, por medio de herramientas Tac, las que tienen un mayor impacto son:

- Registraban notas en el software de Compucol (83\%).

- Registraban calificaciones por medio de Excel (60\%).

- Realizaban evaluaciones utilizando el computador como medio tecnológico para desarrollar y guardar la información (68\%).

- Realizaban evaluaciones según los contenidos del portal Colombia aprende (64\%).

Como se pudo observar, los docentes utilizaban con más frecuencia las tecnologías para registrar las calificaciones de los estudiantes en el sistema institucional y en las herramientas de bases de datos como Excel, y solamente realizaban evaluaciones por medio del computador. En cuanto a las debilidades para evaluar a los estudiantes a partir de las Tac se encontró que los docentes:

- No evaluaban a los estudiantes con ningún software de aplicación de pruebas (66\%).

- No enviaban correcciones de trabajos desde una plataforma personal (66\%).

- No usaban la plataforma educativa para dar feedback a sus estudiantes (75\%).

- Nunca enviaban correcciones a través de herramientas como medio webs 2.0 (65\%).

- No enviaban correcciones de las pruebas por medio del correo electrónico (62\%).

Se pudo apreciar que los docentes no diseñaban ni corregían evaluaciones haciendo uso de las tecnologías presentes para tal fin como Quiz creator, plataformas, webs 2.0 e incluso el correo electrónico para compartir las pruebas aplicadas con sus correspondientes correcciones.

\section{Discusión}

El resultado de la presente investigación en el uso didáctico de las Tac, mostró que éste es deficiente, lo que significó que los docentes hacían un mínimo uso de las Tac en el proceso de enseñanza. Dentro del evento de estudio se trabajaron cuatro dimensiones: planificación, motivación, facilitación y evaluación. La dimensión planificación fue ligeramente mejor que las otras, y se ubicó en la categoría regular, lo que significó que en ocasiones los docentes usaban las Tac en el proceso de enseñanza.

Entre las fortalezas en el uso didáctico, se pudo destacar que los docentes se inclinaban a la búsqueda de información por medio de internet para la explicación de temáticas, y a su vez utilizaban procesadores de textos para la elaboración de talleres, mientras que las debilidades presentadas mostraron que los docentes en su gran mayoría no usaban las tecnologías emergentes para la preparación de los planeamientos de clase, ya que no utilizaban blogs, plataformas educativas, apps, videotutoriales ni software que podrían mejo- 
rar significativamente la preparación de contenidos y actividades.

Ahora bien, el uso didáctico en cuanto a la facilitación mostró que el grupo de docentes les faltaba implementar significativamente actividades mediadas por las tecnologías en el aula de clase. Por otra parte, las fortalezas encontradas fueron que los docentes tenían un buen potencial en el uso del internet para actividades de consulta y apoyaban sus explicaciones por medio de tecnologías básicas como la presentación de diapositivas.

Los docentes todavía no han incorporado tecnologías más recientes dentro de los procesos educativos en las diferentes actividades de su labor educativa. Sin embargo, se pudo percibir que las debilidades de los docentes en el uso didáctico en la dimensión de facilitación estuvieron en la dificultad para usar tecnologías en el desarrollo de actividades junto a sus estudiantes, ya que no usaban herramientas que podrían mejorar la comunicación e interactividad entre docente-estudiante por medio de wikis, blogs, redes sociales e incluso el correo electrónico para compartir información. Además, tecnologías actuales como realidad aumentada no eran incluidas dentro del proceso de enseñanza aprendizaje.

\section{CONCLUSIONES}

Se evidenció que los docentes de básica secundaria y media de las instituciones educativas públicas del municipio de Chiquinquirá, tenían un bajo nivel de uso de las tac en la planeación de los contenidos de las clases, igualmente con la facilitación en el proceso de enseñanza aprendizaje, en la cual el uso de las tac era mínimo en el desarrollo y explicación de las diferentes temáticas que se abordaron en clase, lo cual implicó que, los estudiantes no estuviesen muy involucrados con las tecnologías dentro del aula. De la misma forma la evaluación hacia los estudiantes no presentó cambios ya que se utilizaron con mínima fre- cuencia las tecnologías educativas para evaluar contenidos, eran solo utilizadas para llevar registros de calificaciones. Se pudo establecer que los docentes con excelente uso didáctico de las tac en el proceso de enseñanza aprendizaje eran los docentes de tecnología e informática y de idioma extranjero inglés.

\section{REFERENCIAS}

Arranz, A. 2017. Aprendizaje memorístico: Cuando retenemos datos sin profundizar en su significado. Blog Cognifit Salud, Cerebro \& Neurociencia. Disponible en línea: https://blog.cognifit.com/es/aprendizaje -memoristico/

Bakia, M., Murphy, R., Anderson, K y Trinidad, $G$ (2011). International Experiences with Technology inEducation: Final Report. Washington, D.C. U.S. Department of Education

Bautista, R. 2017. El uso didáctico de las tics en el mejoramiento de la labor didáctica en la escuela colombiana, Aibi revista de investigación, administración e ingeniería, vol. 5, No. 2, pp. 2-8

Cabero, J. 2007. Las necesidades de las TIC en el ámbito educativo: oportunidades, riesgos y necesidades. Revista Tecnología y Comunicación Educativas. Año 21, No. 45. (Pp. 4-19)

Díaz, F. 2013. TIC en el trabajo del aula. Impacto en la planeación didáctica. Revista Iberoamericana de Educación Superior, 4(10). (Pp 3-21)

Fernández, M y Madrid, D. 2010. Modelos Didácticos y estrategias de enseñanza en el espacio Europeo de Educación Superior. Revista: Tendencias Pedagógicas. Volumen 1, No. 15. (Pp. 91-111)

Francesc, P. (2014). Tecnologías para la mejora de la educación: experiencias de éxito y expectativas para el futuro. Fundación Santillana. Recuperado de:https://www. mecd.gob.es/dam/jcr...155b.../docbasico 2015semeducsantillana-pdf.pdf

Fundación Comillas. 2018. Metodología pasiva vs activa. Blog Centro universitario CIESE. Fundación Comillas. Disponible en 
línea:

https://www.fundacioncomillas.es/actua lidad/noticias/view/metodologia-pasivao-activa/

Lozano, R. 2011. De las TIC a las TAC: Tecnologías del aprendizaje y del conocimiento. Revista Anuario ThinkEPI. Volumen 5. (Pp. 45-47)

McLoughlin y Lee (2008), Fecha de consulta: 16/07/2017. Disponible en: http://www.scirp.org/(S(351jmbntvnsjt 1aadkposzje))/reference/ReferencesPap ers.aspx?ReferenceID=198593

MEN. 2009. El ideal educativo del nuevo siglo. Recuperado de: https://www.mineducacion.gov.co/1621/article-209856.html. Consulta: 07/04/2017

Ortega, A. 2014. La importancia de las Tecnologías de Información y Comunicación en la Enseñanza de los Jóvenes del Siglo XXI. Revista Razón y Palabra. Vol.18 N87

Pinzón, F. 2017. Objetos Virtuales de Aprendizaje, una Estrategia Didáctica para el Mejoramiento de los Procesos de Enseñanza Aprendizaje en Zonas Rurales. (Tesis Doctoral). Ciudad de Panamá, Panamá. UMECIT

Quintero, G. 2008. La Facilitación de Procesos de Aprendizaje. Facilitación de Formadores. Caracas, Venezuela. Disponible en: file:///C:/Users/usuario/Downloads/BFacilitaci\%EF\%BF\%BDn-de-Procesos-deAprendizaje.pdf

Rivero y Moscoso. (2013). Planificación Educativa para la atención de niños de 0 a 3 años. Ministerio de Educación de Perú. Disponible en: http://www.ugel07.gob.pe /wpcontent/uploads/2016/04/Guia_pla nificacion_educativa_ciclo1-UGEL07.pdf

Santiago G, Caballero R, Gómez D, Domínguez, A. (2013). El uso didáctico de las TIC en escuelas de educación básica en México. Revista Latinoamericana de Estudios Educativos (México) [en línea] 2013, XLIII [Fecha de consulta: 26 de febrero de 2019] Disponible en:<http://www.reda lyc.org/articulo.oa?id=27028898004> ISSN 0185-1284

Siemens, G. 2004. Connectivism: A Learning Theory for the Digital Age. Documento en línea: Disponible en: www.itdl.org/journal/jan_05/article01.htm. Consulta: $07 / 04 / 2017$

UNESCO. 2009. Medición de las Tecnologías de Información y Comunicación (TIC) en Educación manual del usuario. Canadá. Editorial UNESCO-IEU

UNESCO. 2013. Enfoques Estratégicos sobre las TICs en Educación en América Latina y el Caribe. Chile. Editorial OREALC/ UNES$\mathrm{CO}$

Valdés, Angulo, Nieblas, Zambrano y Arreola. 2012. Actitud de docentes de secundaria hacia el uso de las TIC. Revista Investigación Educativa Duranguense, №. 12, 2012. (Pp. 4-10)

Valenzuela (2015). Motivación escolar: Claves para la formación motivacional de futuros docentes. Revista Estudios Pedagógicos XLI, No 1 (Pp. 351-361)

Viñals, A y Cuenca, J. 2016. "El rol del docente en la era digital". Revista Interuniversitaria de Formación del Profesorado. Volumen 30, No. 2. (Pp. 103-114) 